\title{
Concerns about the interpretation of OCT and fundus findings in COVID-19 patients in recent Lancet publication
}

\author{
Demetrios G. Vavvas $\mathbb{1}^{1,2,3,4} \cdot$ David Sarraf ${ }^{5,6,7,8} \cdot$ SriniVas R. Sadda ${ }^{9,10,11} \cdot$ Dean Eliott ${ }^{1,3} \cdot$ Justis P. Ehlers ${ }^{12}$. \\ Nadia K. Waheed ${ }^{13}$ - Yuki Morizane ${ }^{14}$ - Taiji Sakamoto ${ }^{15} \cdot$ Miltiadis Tsilimbaris $^{16} \cdot$ John B. Miller $^{1,17,18}$
}

Received: 1 July 2020 / Revised: 1 July 2020 / Accepted: 1 July 2020 / Published online: 9 July 2020

(c) The Royal College of Ophthalmologists 2020

We read with great interest the correspondence in Lancet by Marinho et al. [1] describing purported retinal manifestations of coronavirus disease (COVID-19). While there is great interest to understand potential ocular complications of COVID-19 during this pandemic, we have some concerns regarding the interpretation of the fundus and optical coherence tomography (OCT) findings.

Marinho et al. examined 12 COVID-19 patients (all confirmed by PCR or antibody testing) with typical systemic disease but no visual symptoms. The reported cohort was relatively young (25-69 years; no mean or median provided) without severe systemic disease, although 2 were hospitalized. Eleven of the 12 were physicians or health care workers.

The authors reported that 4 of the 12 patients displayed lesions consistent with subtle cotton wool spots (CWSs), i.e., retinal nerve fiber layer (NFL) infarcts, and illustrated one example lesion. It should be noted that CWSs can be

Demetrios G. Vavvas

vavvas@mee.harvard.edu

1 Department of Ophthalmology Retina Service, Harvard Medical School, Boston, MA, USA

2 Ocular Regenerative Medical Institute, Boston, MA, USA

3 Massachusetts Eye and Ear Infirmary, Boston, MA, USA

4 Massachusetts General Hospital, Boston, MA, USA

5 Department of Ophthalmology, Stein Eye Institute at UCLA, Los Angeles, CA, USA

6 Retinal Disorders and Ophthalmic Genetics Division, Los Angeles, CA, USA

7 Pacific Retina Club and International Retinal Imaging Symposium, Los Angeles, CA, USA

8 International Retinal Imaging Society, Los Angeles, CA, USA

9 Doheny Eye Institute, Los Angeles, CA, USA

10 Doheny Image Reading Center (DIRC), Los Angeles, CA, USA identified in a broad spectrum of diseases [2,3] and it is unclear if there were any comorbid conditions that could have confounded this analysis. Furthermore, the lesion illustrated in the report was very subtle and may just represent NFL myelination, which is not an abnormal finding. OCT angiography was reportedly normal indicating that the lesion was not likely pathological. Follow-up evaluation of this lesion is essential. A CWS would be expected to disappear within 6-8 weeks while myelination would remain unchanged. Without such an analysis, it is impossible to know for certain if this is truly a pathological CWS.

Of greater concern, the authors illustrated OCT findings of three patients with hyper-reflective bands in the inner retina that they interpreted as abnormal despite reportedly normal OCT angiography. They localized these findings to the inner plexiform and ganglion cell layers; however, the images provided indicate localization to the ganglion cell layer. These OCT findings bear significant resemblance to

11 Department of Ophthalmology, University of California - Los Angeles (UCLA) Geffen School of Medicine, Los Angeles, CA, USA

12 The Tony and Leona Campane Center for Excellence in ImageGuided Surgery and Advanced Imaging Research, Cole Eye Institute, Cleveland Clinic, Cleveland, $\mathrm{OH}$, USA

13 Boston Image Reading Center and Tufts Medical Center, Boston, MA, USA

14 Department of Ophthalmology, Okayama University, Okayama, Japan

15 Department of Ophthalmology, Kagoshima University, Kagoshima, Japan

16 Department of Ophthalmology, University of Crete, Heraklion, Greece

17 Department of Retinal Imaging, Retina Service, Massachusetts Eye and Ear, Boston, MA, USA

18 Harvard Retinal Imaging Lab, Boston, MA, USA 
Fig. 1 Spectral domain OCT images of a healthy adult from the pre-COVID era showing the appearance of normal vessels in the inner retina similar to the findings reported in the letter by Marinho et al. As noted on the near infrared, these findings correspond to normal retinal vessels. Top panel shows a vessel in oblique cross-section, and bottom panel shows a vessel along its long axis. Green line highlights where the B-scan of the OCT was acquired superimposed automatically by the acquisition instrument on an en face infrared fundus image. White arrows and bracket point to the vessels imaged on the OCT.
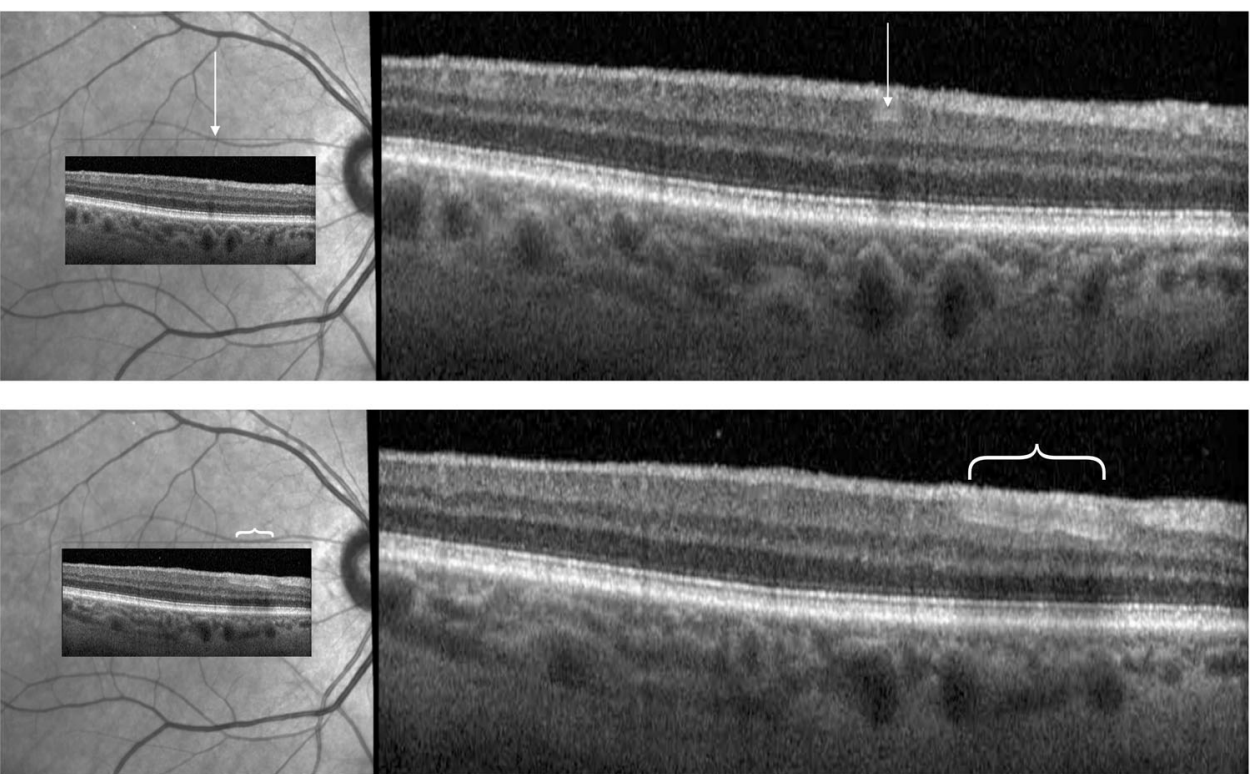

normal inner retinal vessels [4] in terms of the morphology, reflectivity, location, and associated posterior shadowing, characteristic OCT features of blood vessels, as illustrated in an unrelated case in Fig. 1. Thus these reported OCT findings may not be related to COVID-19 and may simply represent normal retinal vessels. This may also be the reason that Marinho et al. reported to have seen them in all patients. The authors did not provide near infrared reflectance (NIR) registration to confirm that the hyper-reflective bands do not represent normal vessels. Initial and follow-up images of the entire volume of OCT scans with the registered NIR images would be essential for accurate interpretation.

While we applaud the authors' efforts to gain insights into this era-defining disease that has affected all aspects of life worldwide, any uncertainty of reports on its manifestations needs to be rectified and clarified. We believe that this additional information for review by the scientific community is of paramount importance.

\section{Compliance with ethical standards}

Conflict of interest The authors declare no conflict of interest.

Publisher's note Springer Nature remains neutral with regard to jurisdictional claims in published maps and institutional affiliations.

\section{References}

1. Marinho PM, Marcos AAA, Romano AC, Nascimento H, Belfort R. Retinal findings in patients with COVID-19. Lancet. 2020. https://doi.org/10.1016/S0140-6736(20)31014-X.

2. Brown GC, Brown MM, Hiller T, Fischer D, Benson WE, Magargal LE. Cotton-wool spots. Retina. 1985;5:206-14.

3. Ioannides A, Georgakarakos ND, Elaroud I, Andreou P. Isolated cotton-wool spots of unknown etiology: management and sequential spectral domain optical coherence tomography documentation. Clin Ophthalmol. 2011;5:1431-3.

4. Lee CS, Tyring AJ, Wu Y, Xiao S, Roken A, DeRuyter NP, et al. Generating retinal flow maps from structural optical coherence tomography with artificial intelligence. Sci Rep. 2019;9:5694. 